\title{
LA UTILIZACION DE LA BIOMASA COMO COMBUSTIBLE RENOVABLE
}

\author{
J. P. VALCARCEL M. y F. VALCARCEL M. *
}

\section{RESUMEN}

La evolución de los diferentes ecosistemas de la región tropical de Colombia ha sido el resultado de la posición geográfica, de la acción de los factores y procesos pedologicos y edafológicos, de la acción climática, tectónica y geológica, de la acción de la micro y macro fauna y en especial de la intervención antrópica sobre los recursos naturales renovables. Algunas de las causas que han llevado, en forma de reacción en cadena, al deterioro del medio ambiente, son: la acción de los fenómenos naturales, la minería no tecnificada, la migración de la población rural a los centros urbanos, la infraestructura vial, la industrialización, la colonización, la utilización de la madera para energía, el sobre pastoreo, los cultivos lícitos e "ilícitos", contaminación de aguas y suelos por el uso de pesticidas y agroquímicos y la ampliación de la frontera agrícola entre otros. La agricultura es la actividad humana que más depende del medio ambiente natural y tiene una relación más estrecha con él. Inevitablemente la agricultura se ha convertido en una de las causas principales de modificación en el medio ambien-

* J.P. Valcárcel es profesor de la Universidad Surcolombiana, Facultad de Educación.

F. Valcárcel es profesor de la Universidad Nacional de Colombia, Facultad de Agronomía, Posgrado en Suelos. te. Aunque los residuos de origen animal han sido utilizados por el hombre desde tiempos remotos, actualmente se presenta una tendencia en virtud de los programas y proyectos nacionales e internacionales a desarrollar una agricultura biológica y sostenible, incrementándose el uso de abonos orgánicos a base de abonos verdes o excretas de animales, bien descompuestas, como la gallinaza, bovinaza, porquinaza y lombrinaza. Por ser estos materiales de rápida biodegrabilidad, muchos son los efectos benéficos producidos por los abonos orgánicos: desarrollo de las plantas, conservación y recuperación de las propiedades físicas del suelo (estructura, retención de humedad), mejoramiento de las propiedades químicas de los suelos (liberación y movilización de nutrimentos como N, P, K, Ca, Mg, $\mathrm{B}, \mathrm{Fe}, \mathrm{Mn}, \mathrm{Zn}$, y $\mathrm{Cu}$ ) y el incremento y presencia de microorganismos que enriquecen y activan la población biológica del suelo. De otro lado, por ser una práctica económica y eficaz el nivel de vida de la población rural se incrementa, llegando a ser autosuficientes con una agricultura sana e industria a pequeña escala. Supliendo de esta manera sus necesidades básicas, presentándose un estilo de desarrollo, nuevas oportunidades de subsistencia y menos daño ambiental.

\section{INTRODUCCIÓN}

El mundo enfrenta serios problemas ambientales: comenzando por la erosión 
hasta drásticos cambios climáticos acompañados por la contaminación, los cuales son conocidos y que muestran graves efectos y consecuencias todavía imprevistas. La Tierra ha estado perdiendo un estimado de 10 a 20 millones de hectáreas de tierra tropical debido a la erosión, a los deslizamientos, a la tala de árboles y a la limpieza inadecuada e incontrolada para los usos agrícolas. Para el año 2000 cerca de 275 millones de hectáreas habrán sido destruídas, contándolas desde el año 1980. La producción sostenible y la conversión de plantas y residuos vegetales como combustibles, ofrecerá un potencial significativo para aliviar la presión que pesa sobre el uso, para el mismo fin, de reservas forestales nativas y selvas. Junto con los desmontes hechos para la agricultura estos apremios y atosigamientos han sido las mayores amenazas para la selva y para los recursos forestales, pantanos y vertientes y ecosistemas de las tierras altas.

Durante los últimos 200 años, la deforestación, la deflagración y el actual consumo de combustibles fósiles han agregado $\mathrm{CO}_{2}$ a la atmósfera, que muestra un incremento en la concentración de $\mathrm{CO}_{2}$ del $27 \%$ (la mitad del incremento que ha ocurrido en los últimos 30 años). El efecto resultante conocido como invernadero ha venido acompañado también de un incremento en la temperatura promedio global de cerca de $0,5{ }^{\circ} \mathrm{C}$ y el nivel total de los mares se ha incrementado de 10 a $20 \mathrm{~cm}$. Si no cambiamos nuestros estilos de vida la temperatura global media podría incrementarse en 0,3 ${ }^{\circ} \mathrm{C} /$ década y el nivel del mar en $6 \mathrm{~cm} /$ década. Sin lugar a dudas, el factor simple más importante en todas estas predicciones es la rata de combustión de los combustibles fósiles. Los combustibles obtenidos de la biomasa no proporcionan ninguna contribución al $\mathrm{CO}_{2}$ atmosférico si se utiliza en forma sostenible y pueden considerarse como una aproximación práctica a la protección del ambiente (calentamiento global) y al sostenimiento de una prevalencia y evolución de los elementos terminales tales como revegetación (incluyendo la reforestación).

Calentamiento de invernadero y sumideros de biomasa para el $\mathrm{CO}_{2}$

La reforestación y la revegetación juegan papeles importantes en la reducción de la contaminación. En 1980 el escape neto de carbón a la atmósfera debido a la deforestación e incendios se mantuvo en el rango de 1 a 2 billones de toneladas (1 billón en $U S A=$ mil millones). Existe amplia evidencia de que la rata de deforestación se ha incrementado substancialmente desde 1980 en varios lugares de los trópicos, aunque no ha habido un estudio más comprensivo e importante de lo que sucede en los trópicos, como un todo, desde ese entonces. En 1980 la rata de deforestación de los bosques tropicales fue de 11,3 millones de hectáreas y entre 18 y 20 millones de hectáreas en 1989. Se presume que una cantidad superior a los 150 millones de hectáreas serán deforestadas en la década de los 90.

Se consideran esencialmente 3 formas para disminuir el uso de combustibles fósiles:

a. mejorando la eficiencia energética.

b. desarrollando fuentes de energías renovables.

c. ampliando la utilización de la energía nuclear.

La protección ambiental y el costo efectivo que esto implica tienen un común denominador: la eficiencia de la conversión de la energía. Entre menor sea la cantidad de combustible usado por kilovatio-hora de electricidad generada, menor será también la cantidad de emisiones de dióxido de carbono y de otros contaminantes. Mientras las reservas sean conservadas, solamente el costo efectivo en la generación de potencia limpia será la orientación mundial para en- 
contrar el uso y el crecimiento en la demanda de energía. La eficiencia energética es una necesidad inmediata pero debe estar acompañada por el desarrollo de las energías renovables. Las energías renovables, como un todo, juegan un papel importante en la reducción de la contaminación, particularmente con el uso de la biomasa como energía. Un esfuerzo gigante de reforestación mundial es una posible estrategia para preservar y expandir las selvas del mundo y disminuir el avance en el cambio climático. A medida que los árboles crecen, ellos capturan el $\mathrm{CO}_{2}$ de la atmósfera y de esa manera disminuyen la producción de $\mathrm{CO}_{2}$. La plantación de árboles en escala masiva y a largo término es uno de los medios más prácticos de remoción del $\mathrm{CO}_{2}$. Una estrategia mundial que conduzca a reducir a la mitad la deforestación tropical y promover la plantación en un equivalente de unas $130 \mathrm{mi}$ llones de hectáreas de árboles en países desarrollados y 40 millones de hectáreas en naciones industrializadas podrían reducir las emisiones de carbón en el mundo como consecuencia de todas las actividades humanas, por un factor de cerca de un cuarto de los niveles corrientes.

Restringiendo el consumo de carbón por un tiempo de 20 a 60 años, mientras crecen los árboles, se podrían estabilizar los niveles de $\mathrm{CO}_{2}$ en la atmósfera y posteriormente, con la utilización de la biomasa para reemplazar los combustibles fósiles, se podría llegar a una disminución notable de $\mathrm{CO}_{2}$. Tal vez una mejor estrategia económicamente posible podría ser la sustitución del combustible fósil, especialmente la del carbón, con combustibles de biomasa. Este estudio mostró que la retención del carbón en bosques es una estrategia relativamente de bajo costo para compensar las emisiones de $\mathrm{CO}_{2}$ por la combustión de combustible fósil y se podrian obtener substancialmente mayores beneficios mediante el sustituto de combustibles fósiles con biomasa, desarrollada en manera sostenible y convertida en ener-

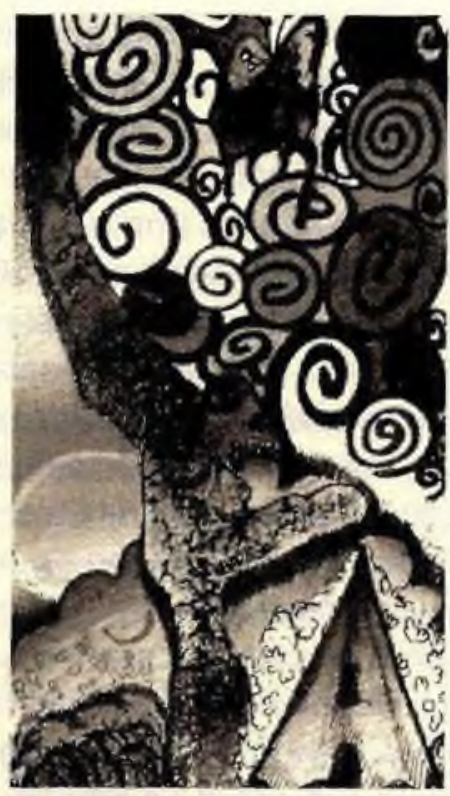

Ilustración: Alexander Garcia Tovar

gía útil utilizando tecnologías modernas de conversión. Esto puede hacerse utilizando gasificación por biomasa empleándolo en turbinas para generar electricidad o por hidrólisis enzimática de biomasa maderable para producir combustibles de alcohol. La biomasa, como substituto del carbón, puede ser tan efectiva como el confinamiento o ahorro en el consumo de éste, por tonelada de biomasa, en la reducción de las emisiones de $\mathrm{CO}_{2}$; no obstante, la substitución del combustible puede llevarse a cabo indefinidamente, mientras que la retención del carbón puede ser efectiva solamente hasta que los bosques alcancen su madurez.

En gran parte los recursos de la biomasa pueden ser considerados un tanto lejanos en la substitución de combustible en cualquier tiempo, más que de la retención del carbón porque, primero, los productores tenderán a mirar las especies de la biomasa con mayores cultivos anuales para aplicaciones energéticas y, segundo, la biomasa para la energía puede ser obtenida de otras fuentes y no únicamente de los nuevos bosques. Por tanto, la biomasa puede jugar un papel muy especial en la reducción del calentamiento de invernadero substituyendo 
el combustible fósil más que por retención del carbón. Además, la energía de la biomasa es potencialmente menos ventajosa que la energía del combustible fósil en un amplio rango de circunstancias, por lo que el costo neto en desalojar las emisiones de $\mathrm{CO}_{2}$ podría ser negativa. Luego las estrategias bioenergéticas tienen "construído" incentivos económicos que hacen de ellas algo más fácil de implementar que otras estrategias con el fin de que tengan la suficiente fuerza para competir con el calentamiento de invernadero. Las estrategias de largo alcance son posibles pero su implementación será extremadamente dificil a menos que existan incentivos substanciales garantizados a largo plazo para la siembra de árboles. Estas estrategias pueden incrementar la plantación de árboles y la revegetación generalmente para dar una óptima remoción de $\mathrm{CO}_{2}$. Además de la remoción del $\mathrm{CO}_{2}$, los bosques proveen otros beneficios complementarios. La plantación de árboles podría mejorar la situación energética proveyendo combustible maderable, suministrando generación de ingresos y podrían tener efectos ecológicos muy importantes asociados con la rehabilitación de la tierra tales como el control de erosión del suelo, el mantenimiento de las vertientes, mejoramiento de los climas locales, la prevención de la destrucción de los humedales y de las áreas altas, etc. Se estima que $\mathbf{2 0 0}$ millones de hectáreas necesitan ser reforestadas por razones diferentes a las del control del efecto invernadero.

Es evidente que la mayoría de beneficios inmediatos se derivarán del incremento en la eficiencia de la energía y en la reducción de la rata de deforestación y de la devastación vegetal. Aunque la detención de la deforestación será muy difícil, ésta parece ser la mejor opción en corto término. Pero es esencial entender el uso actual de la biomasa antes de planificar la revegetación y la detención de la deforestación (de otra manera no serán de mucha ayuda los pla- nes bien intencionados). La producción de biomasa bien sea en condiciones naturales o con bosques plantados, lotes maderables o árboles dispersos necesita ser optimizado en su ambiente de manera sostenible. Ya se han considerado temas tales como la optimización de los campos con policultivos en lugar de monocultivos para asegurar alguna biodiversidad, intercalando especies fijadas con nitrógeno de manera que se disminuya la absorción y filtración de fertilizantes, el uso de condiciones de optimización de nutrientes para fomentar el empleo de especies existentes y clones. Se considera la conveniencia de obtener altos niveles de diversidad biológica los cuales requieren mantener alguna porción de tierra en regiones de producción de biomasa en una condición "natural". Por ejemplo, algunas especies de pájaros requieren madera muerta y de la población de insectos asociados para su supervivencia. Las experiencias en los bosques de Suecia sugieren que el mantenimiento de una fracción relativamente modesta de un área forestal en tales reservas naturales es adecuada para mantener un alto nivel de diversidad de especies. Los estudios sobre poblaciones de aves en pequeñas rotaciones de bosques en Irlanda muestran que tales plantaciones pueden tener un efecto favorable especialmente donde se han plantado diferentes especies de árboles, se practica la poda y donde progresan numerosas márgenes más que grandes bloques sólidos de clones sencillos o especies. Es necesario continuar las investigaciones para entender qué es lo mejor para obtener los niveles necesarios de diversidad biológica en un amplio rango de condiciones bajo los cuales la biomasa pueda crecer para desarrollar energía en el futuro.

Ya que son mayores los esfuerzos de investigación relacionados con la producción a gran escala de biomasa sostenible, hay tiempo necesario para la investigación e intentos extensivos debido a que las grandes industrias bioenergéticas pueden ponerse 
en operación en las décadas futuras inmediatas utilizando como fuentes primarias las provisiones de residuos alimentarios de los productos industriales agrícolas y de bosques. Tal utilización de residuos así como su monitoreo puede hacerse de una manera aceptablemente ambiental, especialmente de los suelos, para que los nutrientes minerales y los efluentes orgánicos intratables sean devueltos al sitio de crecimiento. Esto se hace, por ejemplo, en la industria del etanol de la caña de azúcar donde los efluentes de fermentación diluídos en el agua de irrigación y regresados a los campos. Se deberían desarrollar prácticas similares como rutinas normales de manejo para que la remoción de residuos, de la agricultura y bosques, sea tenido en cuenta en la producción de energía.

La quema de biomasa bien sea en el hogar o fuera de él puede tener efectos perjudiciales que es necesario reconocer y mejorar. Esto es especialmente serio con fuegos abiertos, en situaciones domésticas encerradas donde surgen problemas para los ojos y los pulmones. El mejoramiento de las estufas de biomasa que reducen las emisiones y aumentan la eficiencia del combustible son objetivos que deben tenerse en cuenta en forma paralela. Afortunadamente la biomasa es un combustible de bajo contenido de sulfuro y también produce menor $\mathrm{NO}_{x}$ que los combustibles fósiles. Estos atributos, combinados con su gran reactividad termoquímica, hacen de la biomasa un combustible atractivo especialmente comparado con el carbón. La quema de árboles, arbustos y pastos producen emisiones de los así llamados gases de invernadero tales como $\mathrm{CO}_{2}$ (principalmente), $\mathrm{NO}_{\mathrm{x}}, \mathrm{CO}, \mathrm{CH}_{4}$ y otros gases residuales. Grandes áreas dé bosques y pastos tropicales son deliberada $y$ accidentalmente quemados anualmente, agregándole a ello el uso extensivo de la biomasa como combustible. La eventualidad de la quema de la mencionada biomasa en el efecto inducido de invernadero solo ha sido reconocida en los años recientes, pero ahora mismo está siendo investigada de forma exhaustiva. Tales quemas no solamente contaminan la atmósfera con el correspondiente incremento de $\mathrm{CO}_{2}$ en la atmósfera (a menos que la biomasa se equilibre por regeneración), sino que puede tener efectos de erosión si los ecosistemas son quemados muy frecuentemente y sobreutilizados por el hombre y los animales de manera que la abrasión del suelo y otros problemas resultarán ser endémicos (mal que se repite constantemente).

\section{Problemas sociales.}

La mayor parte de las discusiones sobre asuntos sociales alrededor de la producción de biomasa y su uso para energía se ha concentrado sobre los problemas y oportunidades en los países desarrollados. Ultimamente y como resultado del incremento de biomasa en los países desarrollados, la agricultura cambiante y las políticas forestales, se han venido dando serias reflexiones al eslabonamiento entre las prioridades socio-

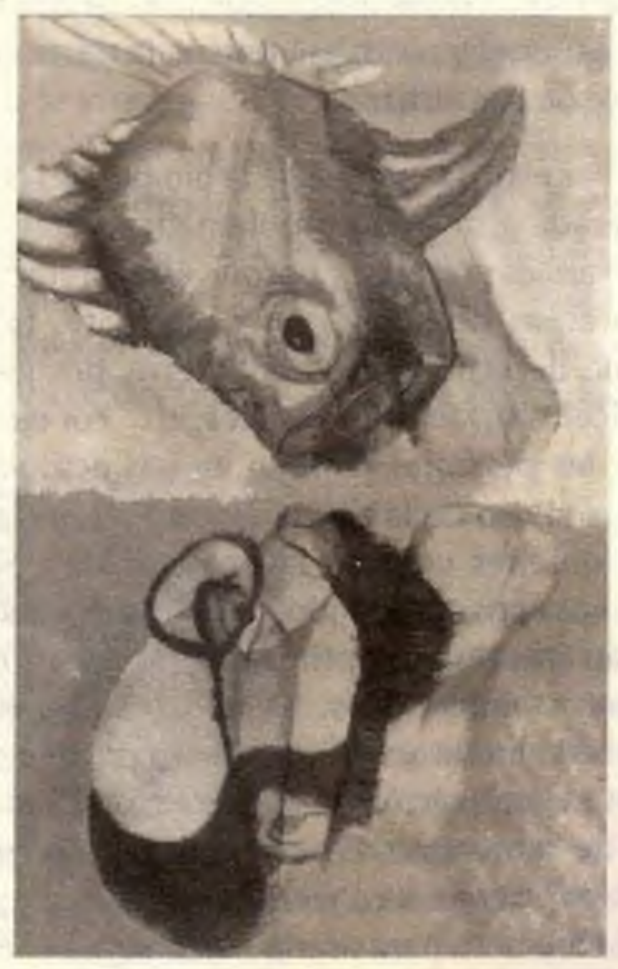

Ilustración: Alexander Carcía Tovar 
económicas y ambientales y el uso de la tierra. Los temas en los países desarrollados y en desarrollo son usualmente considerados como totalmente diferentes, pero ahora ellos convergen en gran medida sobre los problemas de las políticas de utilización de la tierra, subsidios y el ambiente. Por ejemplo, los subsidios para la agricultura en Norteamérica, Europa y Japón sumaron más de 200 billones por año ( 299 billones en 1990) mientras que los subsidios para la energía en USA han sido estimados en 44 billones por año o algo más, caso en el que todas las externalidades escondidas se hayan considerado. Estas cosas distorsionan en gran medida los patrones de uso de la tierra y de la energía. La tierra en excedente para la agricultura en la C.E., se espera que alcance los 15 millones de hectáreas en unos pocos años mientras que en USA cerca de 30 millones de hectáreas están ahora "intocables" bajo los Programas de Conservación de Reservas. La biomasa difiere fundamentalmente de otras formas de energía porque ésta necesita de tierra para crecer o desarrollarse y está sujeta por esa razón al rango de factores independientes utilizados por quienes y cómo gobiernan la tierra que ha se de ser utilizada.

Por esto, la energía de la biomasa es considerada a menudo problemática debido a sus variadas facetas, y porque ésta interactúa con áreas de interés muy diferente, tales como derecho al uso de la tierra, bosques, agricultura, factores sociales, etc. Por ejemplo, las personas difieren en sus actitudes hacia el uso de la tierra: en un extremo están quienes colocan la explotación de la biomasa por sobre todo, mientras que otros están interesados principalmente con los asuntos ambientales. Existen básicamente 2 acercamientos principales para decidir sobre la utilización de la tierra para la energía de la biomasa. El acercamiento "tecnocrático" tiende a concentrarse sobre el uso de la biomasa para usarla únicamente como energía, ignorando otros usos múltiples de la biomasa. Este acercamiento procede de una necesidad por la energía, luego identifica una fuente biológica, el sitio para desarrollarla y luego considera los posibles impactos ambientales. Este acercamiento generalmente ignora muchos de los efectos locales y muchos más de los efectos laterales de las plantaciones para la producción de energía de biomasa ; también ignora las habilidades de los granjeros locales quienes conocen mejor las condiciones locales. Las aproximaciones tecnocráticas han resultado proyectos fallidos en el pasado.

El segundo acercamiento puede ser definido como la aproximación "multiusos" la cual indaga cómo puede hacerse mejor uso de la tierra para un desarrollo sostenible y considera que la combinación del uso de la tierra y los patrones de cosecha harán un uso óptimo de un espacio particular de tierra para encontrar objetivos múltiples, es decir, comida, combustible, forraje, necesidades sociales, etc. Esto requiere de un entendimiento completo de la complejidad del uso de la tierra. Ya que la tierra para la producción de biomasa para energía está tan asediada como para la producción de alimentos y de protección ambiental estos aspectos no pueden ser tratados separadamente. El tema alimento vs combustible ha sido un tópico debatido muy acaloradamente. Para mucha gente la producción de combustible por las cosechas tiene una connotación moral fuerte que sirve para hacer de este aspecto algo muy polémico o controversial. Este aspecto es mucho más complejo de lo que se mostró en el pasado $y$ es el que necesita un examen cuidadoso, ya que las políticas para la agricultura y políticas de exportación y de politización de la adquisición de comida son los factores mayormente determinantes.

Comida vs combustible debería ser analizado al lado de la experiencia de una situación real de alimentación mundial (el incremento de los sobrantes alimenticios en 
un gran número de países en desarrollo y en la mayoría de industrializados) relacionado con la considerable producción de alimentos para animales, el incremento potencial para la productividad agrícola y las ventajas y desventajas de producción de biocombustibles como parte de los múltiples beneficios del uso de la tierra. Es importante apreciar, sin embargo, que la mayor parte de los países en desarrollo están enfrentando problemas tanto de comida como de combustible. Es necesario estimular activamente las prácticas agrícolas para tener presente esta realidad y para desarrollar métodos eficientes de utilización de la tierra disponible y de otros recursos para hallar comida y necesidades de combustible, así como otros productos y beneficios de la biomasa.

Brasil es un caso interesante. La escasez de comida y el aumento de los precios que este país afrontó hace unos pocos años fue censurado por el Programa Proalcohol para la producción de alcohol como combustible. Empero, un examen más estrecho no apoya el punto de vista que la producción de bioetanol haya afectado adversamente la producción de alimentos. La raíz del "problema" reposa profundamente en las políticas económicas del gobierno en general y en la política agrícola en particular. La escasez de alimentos y el incremento de precios en Brasil fueron el resultado de una combinación de procedimientos los cuales esta- ban predispuestos hacia la exportación cómoda de alimentos $y$ al incremento de grandes cantidades de acres de tales cosechas, hiperinflación, devaluaciones monetarias, control de precios a los productos alimenticios domésticos, etc. Dentro de esta realidad cualquier efecto negativo que la producción de biomasa pueda tener deberá considerarse como una parte del problema general, pero no el problema. En realidad, muchas ventajas nacionales y locales se han acumulado con este programa, pero no está indudablemente libre de defectos.

Las plantaciones de biomasa, especialmente las plantaciones de eucaliptos, han recibido críticas adversas en países como India y Tailandia. La mayoría de estas plantaciones no han crecido para ser convertidas a combustible sino para otros usos industriales tales como la pulpa y la construcción. Hay una historia compleja detrás de las experiencias obtenidas en la India y Tailandia la cual no condena al eucalipto como una especie villana, sino que considera los éxitos del eucalipto logrados en Etiopía, Brasil, Portugal, Hawai, etc. La experiencia ha mostrado que las plantaciones para la energía de biomasa son probables de establecer en gran escala en muchos países desarrollados, especialmente en áreas rurales, así como también la siembra de los biocombustibles (particularmente madera) que pueden ser obtenidos a un costo de cero o aproximadamente cero. Los hacendados

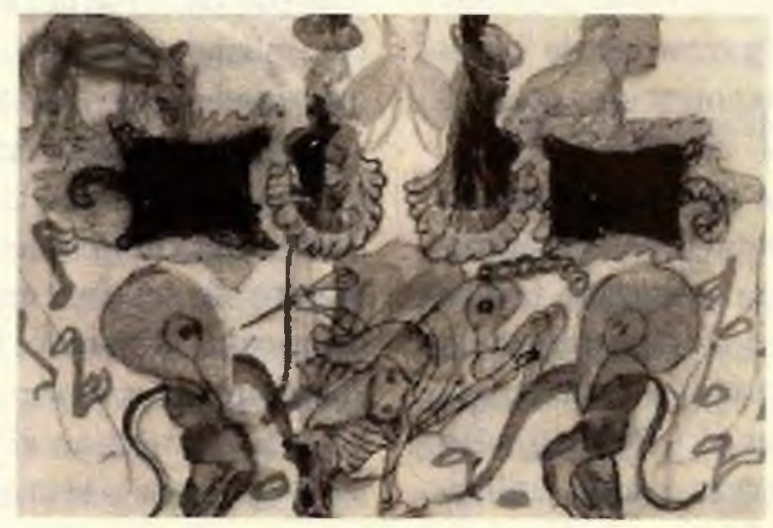

Ilustración: Efren Mosquera Villarreal 
en Malawi, por ejemplo, simplemente no están interesados en los árboles para combustible ya que ellos pueden conseguir grandes ingresos labores y monetarios mediante la plantación de otras cosechas. La modernización de la producción y uso de la bioenergía podría traer beneficios sociales y económicos muy significativos tanto para áreas urbanos como rurales. La falta de acceso a una razonable cantidad de energía, particularmente de transportadores de energía como la electricidad y los combustibles líquidos, limitan de la cantidad de vida de muchos cientos de millones de gente a través de todo el mundo.

Ya que la biomasa es la única fuente de energía más importante en las áreas rurales de los países en desarrollo ésta podría usarse para abastecer las necesidades modernas de energía, por ejemplo, de la agroindustria, bombas para riego, refrigeración, alumbrado, etc.

Además, los sistemas de energía de biomasa deberían ser reconocidos como abastecedores substanciales del intercambio externo si ellos reemplazan los productos del petróleo importado, aunque el asunto no siempre está bien delineado ya que ésto depende en las substituciones de las importaciones y las ganancias de exportación.

En países como Brasil, con una larga experiencia histórica con tecnología para la producción y uso de bioetanol, existen substanciales ahorros en las importaciones de petróleo y también ganancias de intercambio exterior de las exportaciones tecnológicas relacionadas con el alcohol. Zimbabwe similarmente ahorra cambio extranjero sobre las importaciones de petróleo mientras desarrolla la estructura tecnológica que lo conduzca a la importación de substitutos. Así que también tenemos la necesidad de considerar el beneficio neto para un país si las reservas locales utilizadas para la producción de energía domésti- ca pudiesen ayudar a una mayor economía de intercambio extranjero a través de las exportaciones. A pesar del hecho que una gran escala de organizaciones han sido creadas en los países en desarrollo para promover el fomento de la producción a pequeña escala lo cual es especialmente relevante a la energía de biomasa, tales instituciones generalmente no han sido muy efectivas. Muchos gobiernos y proyectos tienden a mantenerse interesados principalmente en la maximización de la salida más que en la economía de capital y la generación de empleo. Los proyectos de alta tecnología reciben prioridad por razones políticas y de prestigio. Cualquier proyecto que falle en el que se reflejen estos objetivos claves que prueban los métodos de capital intensivo, se le reconoce como una demostración de inferior calidad. Estos factores inevitablemente resultan en que mayor cantidad de proyectos relacionados con energía de biomasa reciban baja prioridad o les hagan implementaciones inefectivas.

Por ejemplo, en India, a pesar del interés oficial en el desarrollo de industrias a pequeña escala, la dirección de los negocios públicos y de inversión ha promovido generalmente métodos de producción en gran escala y de grandes inversiones lo cual se muestra inmediatamente en el sector energético. Así que aunque la eficiencia energética y la generación energética de la biomasa relacionada aparezca efectivamente mucho más costosa que la generación de potencia centralizada, esta última es la que recibe la prioridad de los fondos públicos. Retórica y realidad parecen estar desarticulados. El éxito y el fracaso en la provisión de energía de biomasa depende mucho del entendimiento de los incentivos locales y de los obstáculos al cambio. Muchas innovaciones son empujadas dentro de la campaña debido a que interesan al introductor más que las respuestas a las necesidades básicas de la gente a la que ellos tratan de ayudar. Otra 
vez, esta es una actitud especialmente perjudicial hacia los proyectos de biomasa los cuales requieren planeamiento muy cuidadoso y cumplimiento a largo término, lo cual asímismo da múltiples beneficios. Hay muchos otros aspectos sociales los cuales tienen que ver con la energía de la biomasa. Algunos se relacionan con lo anterior: empleo local, oportunidades para los empresarios y desarrollo de habilidades, estabilidad rural sobre una base óptima ambiental, control local de las reservas y la promoción de infraestructuras políticas y económicas apropiadas. La evolución de instituciones y un planteamiento integrado del uso de la tierra el cual abarque la dimensión de la biomasa parecen esenciales si la biomasa no se mantiene por siempre con la alusión a rural pobre y no con la preferencia ambiental de un combustible moderno.

En países desarrollados los intereses y problemas de la energía de biomasa se generan alrededor de riesgos e incentivos económicos del uso de la tierra, superávit de comida, excedentes de tierra, estrategias de reducción de la contaminación, aceptabilidad ambiental, estrategias para las energías renovables y nuclear, alternativa fósil, políticas de forestación, políticas de investigación, etc.

\section{CONCLUSIONES}

¿La biomasa es para siempre?. Es más cierto en el mundo así como lo conocemos, pero puede ser debatido si ésta siguiera para siempre siendo una fuente de combustible. Con una proporción en el aumento de la población mundial que reside en los países en desarrollo, quienes usualmente padecen de combustibles fósiles y de los medios fáciles para importar tales combustibles, es necesario que se ponga un gran esfuerzo para la producción y utilización eficiente de la biomasa como un combustible ya que éste es recurso energético obtenible natural o nativo, el cual puede mejorarse en todas las etapas de producción y conversión.
Uno de los problemas de más amplia aceptación de un ùso moderno para la biomasa en combustibles líquidos, electricidad y gases, en adición a su amplio uso tradicional como fuente de calor, es que éste involucra problemas del uso de la tierra los cuales harán muy difícil su implementación comparado con otras fuentes de energía más centralizadas. Existe un enorme potencial de biomasa no tenido en cuenta, particularmente en la utilización mejorada de bosques existentes y de otros recursos terrestres (incluyendo los residuos) y en la alta productividad de las plantas. Aún más, el mejoramiento en la disponibilidad de biomasa (sobre una base sostenible) requerirá de un considerable esfuerzo (no hay restricción a planes de largo plazo y desarrollo en el campo de la biomasa).

Se necesita que se reconozca que la biomasa está usándose como una fuente de energía no solamente para cocinar en las casas de familia, en muchas instituciones y servicios industriales, sino también en procesos agrícolas en la construcción de ladrillos, tejas, cemento, fertilizantes, etc. Estos usos no culinarios son muchas veces substanciales, especialmente internamente y por todos lados en los pueblos y ciudades. Las poblaciones rurales e industrias de pequeño tamaño están propulsadas con frecuencia por la energía de la biomasa y ésta juega un papel significativo en las economías rurales y nacional; ejemplo, en India estas industrias responden por casi la mitad del sector manufacturero.

\section{Usos modernos de la biomasa}

La desmerecida reputación de la energía de la biomasa como un combustible de pobre calidad, que tiene un pequeño espacio en una economía moderna desarrollada no puede estar más lejos de la verdad. La biomasa debería estar considerada como un equivalente renovable al combustible fósil; ésta puede ser convertida a combustible lí- 
quido vía etanol o a electricidad vía turbinas de gas. Puede también llegar a ser la base de una industria química moderna vía síntesis del gas o etanol como está ocurriendo en Brasil. La biomasa puede servir como abastecimiento para combustión directa en artificios modernos y es mucho más fácil de mejorarla debido a su bajo contenido de sulfuro y a su alta reactividad termoquímica.

Los diversos artefactos o medios de conversión se clasifican desde los más pequeños, marmitas domésticas, estufas y hornos hasta las más grandes calderas y aún a plantas de potencia multimegavatios de gran tamaño. La más amplia explotación comercial sobre una base sostenible espera el desarrollo y aplicación de la tecnología moderna para habilitar la biomasa como competidora con los transportadores energéticos convencionales. Existe un creciente reconocimiento de que el uso de la energía de la biomasa en los grandes sistemas comerciales basados en residuos y actuales recursos sostenibles acumulables se puede favorecer con el fin de mejorar el manejo de los recursos naturales. Si la bioenergía fuera modernizada (es decir, se aplique la tecnología avanzada a los procesos de conversión de biomasa en bruto en transportadores energéticos modernos, fáciles de utilizar tales como electricidad, combustibles líquidos o gaseosos o combustibles sólidos procesados), mucha más energía útil se obtendría de la biomasa de lo que hay ahora, aún sin incrementar los suministros bioenergéticos primarios. En circunstancias favorables, la generación de potencia por biomasa podría ser significativa dadas las inmensas cantidades de silvicultura existente y de residuos agrícolas (por encima de 2 billones de toneladas por año, en el mundo). Por ejemplo, determinados estudios en la industria azucarera y de la industria de pulpa para papel indican una potencia combinada con una capacidad de salida que supera los 500 Terawatios hora/año. (Tera=1012). Asumiendo que una tercera parte de los re- cursos residuales del mundo podrían económica y sosteniblemente ser recuperados por la nueva tecnología energética, se podría generar el $10 \%$ de la demanda eléctrica mundial (10.000 Terawatios hora/año). Además, un programa con un plan de $100 \mathrm{mi}-$ llones de hectáreas sembradas podría también suministrar más del $30 \%$ de la demanda presente mundial. Muchos de los esfuerzos dirigidos a la modernización de la biomasa podrían comenzar con aplicaciones para las cuales los análisis económicos indiquen que son prospectos favorables para un mercado más ágil, o sea, con la generación de electricidad a partir del bagazo de la caña de azúcar, así como los combustibles de alcohol del bagazo de la caña de azúcar y la producción de electricidad usando turbinas modernas de gas operadas por biomasa gasificada obtenida de diferentes fuentes de abastecimiento.

Si los sistemas de energía de biomasa están bien administrados, estos pueden formar parte de una matriz de abastecimiento de energía lo cual resulta ambientalmente óptimo y asimismo contribuya a un desarrollo sostenible. Cuando es comparado, por ejemplo, con los combustibles fósiles convencionales, el gran total en el impacto de los sistemas de bioenergía puede ser menos nocivo al ambiente, ya que ellos producen señales muy localizadas y relativamente pequeñas sobre el circumambiente, comparadas con muy pocos pero más grandes y de mayor importancia de los combustibles fósiles. Son estas las cualidades que hacen que los impactos ambientales producidos por los sistemas de energía de biomasa sean más controlables, más reversibles y, en consecuencia, más benignos. Pero la energía de la biomasa todavía enfrenta muchas barreras: económicas, sociales, institucionales y técnicas. Las fuentes de energía de biomasa son muy amplias y variadas en la naturaleza y las tecnologías para su explotación se extienden a muy diversos rangos en términos de escala, etapas de desarrollo y evolu- 
ción de las exigencias para ser capaces de suministrar un entendimiento bien razonable del problema.

Mientras que el uso tradicional de la energía de la biomasa ha estado siempre con nosotros, la oportunidad para el futuro está concentrada sobre justificaciones más económicas y sobre un óptimo impacto ambiental. Los sistemas avanzados de energía de biomasa deben asegurar al mismo tiempo la producción tradicional y el uso tan eficiente y sostenible como sea posible.

\section{BIBLIOGRAFÍA}

1. Burbano O. Hernán. 1994. La materia orgánica del suelo en el contexto de una agricultura sostenible. Fertilidad de suelos, diagnóstico y control. Sociedad colombiana de la ciencia del suelo. Santafé de Bogotá D. C. pp. 187-217

2. D.O. Hall. October 1991. Energy Policy.

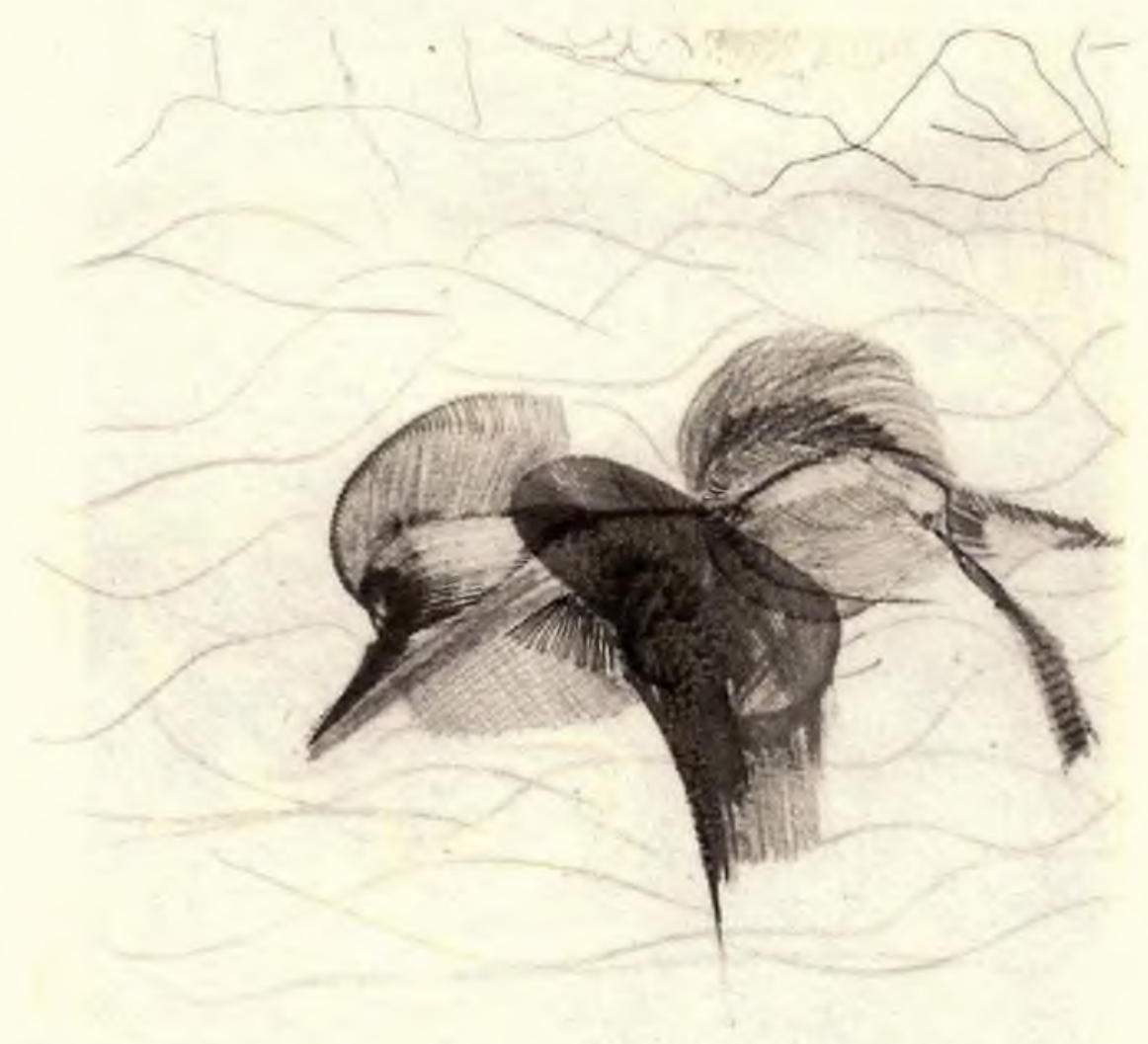

Ilustración: Maribel Castro 